\title{
Efficacy of Sofosbuvir Velpatsavir Combination in HCV Genotype 3 Infected Children of Age between 12 to 18 Years
}

Jawad Khan ${ }^{1 *}$ and Saira Nasr Malik ${ }^{2}$

${ }^{1}$ Department of Gastroenterology, Peshawar Medical College, Kuwait Teaching Hospital, Pakistan

${ }^{2}$ Department of Hematology, Peshawar Medical College, Kuwait Teaching Hospital, Pakistan

*Corresponding Author: Jawad Khan, Department of Gastroenterology, Peshawar Medical College, Kuwait Teaching Hospital, Pakistan.

Received: September 23, 2019; Published: November 04, 2019

DOI: $10.31080 /$ ASGIS.2019.02.0093

\begin{abstract}
Background: very little data is available on efficacy of sofosbuvir(400mg)/velpatasvir(100mg) combination in management of HCV in patients of age between 12 and 18 years.

Aims and Objectives: To determine the safety and efficacy of sofosbuvir(400mg)/velpatasvir(100mg) combination in children between ages 12 to 18 years old with hepatitis genotype 3 infection, the predominant genotype in our part of region.

Methods: This is pilot prospective single arm observational study, 26 patients having HCV infection between age 12 and 18 years were given sofosbuvir/velpatasvir combination for 3 months, during this time various hematological and biochemical investigations and tests were done and measured at baseline (before the start of treatment), at $1^{\text {st }}$ and $2^{\text {nd }}$ months, at end of treatment and after 3 months of treatment. ETR (end treatment response) and SVR (sustained virological response) in form of qualitative PCR were also done to look for response of treatment.

Results: Out of total 26 patients, 3 were lost to follow up after achieving ETR, while all remaining 23 Patients who followed protocol as advised and follow-up visits cured by achieving SVR12 with normal hepatic function, hematological and kidney function tests and no considerable adverse effects.

Conclusion: Our study shows that fixed dose combination of sof/velpa is almost without any side effects and highly efficacious treatment option in children and adolescent between 12 to 18 years who are having genotype 3 infection. Our study should encourage larger studies in this age group in our region where HCV prevalence is one of the highest one in world.

Keywords: Chronic Hepatitis C; Adolescents; Sofosbuvir; Velpatasvir; End Treatment Response; Sustained Virological Response; Safety; Efficacy
\end{abstract}

\section{Introduction}

Hepatitis $\mathrm{C}$ is one of the major virus infection that causes disease of liver in the world almost infecting every corner of the world, currently more than 72 million are suffering from it [1]. Parts of Africa (5.3\%) has highest prevalence in the world, followed by the Mediterranean region in Eastern Europe (4.6\%), regions of Western Pacific (3.9\%) and Eastern Asia (2.15\%) regions. If it comes to the prevalence of hepatitis C in children it is about $0.05 \%$ to $0.36 \%$ in the USA and Europe while in third world countries like Pakistan, India and Bangladesh it is between $1.8 \%$ to $5.8 \%$ [2].
However, this is just the tip of iceberg in this age group and likely underestimate the real number of patients infected with it since patients usually have no symptoms and most of patients don't have easy access to health care facilities, so only a small number of patients having HCV infection can be confirmed having HCV $[3,4]$. New and latest management options are now easily accessible for HCV patients, thanks to an enhanced knowledge of the pathophysiology of the HCV infection, and because with the passage of time new tools have been available for diagnosis and prognostication of disease and advancement in treatment option in form of oral drugs 
which are highly affective as compared to interferon previously and prevention [4].

Pakistan has one of the highest prevalence of HCV in the world currently ranked as $2^{\text {nd }}$ in whole world ( $4 \%$ to $8 \%$ ), while the global prevalence is about $2 \%$. Still, in some regions of Pakistan like Khyber pukhtoonkhwa and Punjab rural areas and Sindh, there are some pocket of areas where the prevalence of hepatitis $\mathrm{C}$ is more than $20 \%$ [5].

It is estimated that the number of HCV infected children world widely is about 5 million, aged 12-18 years. The main route of infection is mother to infant, but there are a lot of other possible sources of infection, like nosocomial infection. IV drug abuse is one of the major source of infection in adults. The epidemic of IV drugs in the US two decades ago did showed that infected needles can be a huge source of infection from mother to her children. The transmission rates are higher from HIV-HCV coinfection as compared to HCV only. So it is recommended that all children whose mothers are HCV-infected should be tested for HCV infection once they cross 18 months because below 18 months the presence of maternal antibodies in infants makes the test unreliable. Cirrhosis and Hepatocellular carcinoma are usually rare in children, but they can occur [6]. Sometimes, complications of HCV do occur during early life [7]. patients infected with thalassemia iron overload, HIV HCV coinfection and hematological or tumors receiving chemotherapy, may develop advanced liver diseases and complications when infected with HCV too [8]. Obesity may be one of the cause for advance liver disease due to synergistic affect. Limited number of treatment options are available for children below 18 years especially for genotype 3 . Recently some clinical trials have shown that Direct antiviral drug based regimens are very effective in children and adolescents like sofosbuvir/ledispevir in genotype 1,4,5,6 and other is sofosbuvir/ribavirin in genotype 2 and 3 . So sofosbuvir/ ledipasvir is approved for genotypes 1, 4, 5 and 6 and sofosbuvir/ ribavirin for genotypes 2 and 3 for adolescents aged 12-17 years, or body weight greater than $35 \mathrm{~kg}$, having HCV [9].

In a developing country like Pakistan with the one of the highest prevalence of chronic HCV infection, health authorities are very keen and working very hard to control and tackle HCV and has introduced multiple treatment programs all over the country which is absolutely free of cost, easily accessible, following world health organization (WHO) roadmap, to eliminate hepatitis $\mathrm{C}$ by the year 2030; to achieve this goal in real, economic and cost effective strategies are important to sustain and continue this program affectively. To achieve this goal, health policy makers would adapt the international guidelines for treatment of chronic HCV and prevention strategies. Availability of low-cost, generic, pan-genotypic drugs has made possible to provide cost-effective strategy, enabling the government to pursue its important program in efficient way through the great reduction in price of the drug. Also there is no need of genotype testing as it is very expensive and available in very few centers, making this more cost effective and affordable. Fortunately generic drugs are available which are very cheap and easily accessible all over the country.So keeping this mind, We conducted this study to investigate the side effect profile and effectiveness of the pan genotypic sofosbuvir/velpatasvir therapy in Pakistani people between age 12 to 18 infected with chronic HCV infection.

The predominant genotype in Pakistan is genotype 3(70\%) [10]. The only approved combination for these 2 genotypes is combination of sofosbuvir and ribavirin in children between 12 to 17 years age. It is associated with comparatively low response rate specially in genotype 3 which is the hardest to treat and keeping in mind the side effects of ribavirin, an alternative and more affective option is needed in this group with low side effect profile. Sof/velpa is pan genotypic combination with very low side effect profile and SVR more than $95 \%$ in genotype 3 make it a realistic choice for it, so we tested this combination in genotype 3 patients of age between 12 to 18 for SVR and side effect profile.

\section{Material and Methods}

This study was carried out as a single arm prospective pilot observational single center study on efficacy of sofosbuvir/velpetasvir in management of HCV infection between 12- to 18-year old children presenting to Gastroenterology Department, Services Hospital Peshawar during the period from January 2018 to December 2018. Inclusion and exclusion criteria is given below. 


\section{Inclusion criteria}

- $\quad$ Age: 12 - to 18 - years.

- $\quad$ Confirmed diagnosis of chronic HCV infection with positive qualitative PCR cut off more than $15 \mathrm{iu} / \mathrm{ml}$

- $\quad$ HCV genotype $3 a$ and $3 b$.

- $\quad$ AST to platelet ratio (APRI) score less than 2.

- Weight more than $35 \mathrm{~kg}$.

\section{Exclusion criteria}

1. Liver disease other than HCV.

2. HIV positive patient.

3. ALT or AST more than 3 times.

4. APRI score more than 2 indicating advance fibrosis.

5. Platelets count $<50000$.

6. Hemoglobin less than $8 \mathrm{gm} / \mathrm{dl}$.

7. Parents' refusal.

The study protocol was approved by the ethical Committee of services hospital, proper consent with full information ware taken from patients' parents for including their children in the study and for publication, data starting from January 2018 to December 2018, patients presenting, with chronic HCV infection with genotype 3 , fulfilling the inclusion criteria mentioned above. The 26 patients having fulfilling eligibility criteria with normal liver functions were included in this study as a single treatment group. During the screening baseline visit (week 0) and all other study visits (month 1, month 2, month 3) End Treatment response (ETR) and month 6 sustained virological response (SVR), full physical examination and investigations like the complete blood count (CBC), serum bilirubin, serum albumin, Alanine aminotransferase (ALT) and Aspartate aminotransferase (AST), APRI, serum creatinine and ultrasound of abdomen were done on included patients. The serum HCV-RNA was tested using the Polymerase Chain Reaction (PCR) qualitative measurements for diagnosis, for ETR and SVR.

All patients were frequently asked and examined for any side effects during the study they were also given contact numbers to report any adverse effect. All patients were given daily single doses of Sofosbuvir $400 \mathrm{mg}$ plus velpetasvir $100 \mathrm{mg}$ combination for 3 months.
Treatment safety was monitored having clinical examination and biochemical and hematological tests during treatment at month 0 , month 1 , month 2 , month 3(ETR), and month 6 (SVR). Close Clinical examination and observation was done at every visit; with any side effect (minor or major) was documented for each patient. Major side effects were defined as events that resulted in stoppage of treatment or death, were noted. Management failure was defined as either: (1) non responder (at end of treatment), or (2) virologic relapse (during follow-up).

\section{Statistics}

Qualitative data were presented as counts, proportions or percentages with the $95 \%$ CI. For quantitative data, descriptive statistics are the arithmetic mean, the standard deviation, mean and median and the 95\% CI whenever found appropriate.

\section{Results}

Initially, 26 patients with full the criteria and were inducted in the study. After managing sustain virological response, 3 patients were lost to follow up so they were not included in study so finally 23 patients concluded study in this study, 6 patients were female and 17 were male the average age was 15 years(SD1.56+_) All the patients were having genotype 3 and qualitative PCR was positive before the start of treatment. All patients were treatment naïve.

There CBC, renal and liver functions were normal at start of treatment, at $1^{\text {st }}, 2^{\text {nd }}$ and at $3 \mathrm{rd}$ month and at SVR. Cirrhosis was ruled out by doing ultrasound calculating APRI score and score was less than 2 for all patients.

Drugs throughout the 3 months was well tolerated with no treatment interruption or death. Minor adverse effects like headache, fatigue and diarrhea did occurred which hardly had an impact on treatment goals. All patients achieved ETR and SVR.

The intention-to treat (ITT) Sustained virological response rate was $23 / 26$, $88 \%$ (95\% CI). The 3 patients lost to follow-up after achieving end treatment response. There was no treatment failure or relapse and all 23 patients (100\%, 95\% CI) who completed the therapy with full duration according to given protocol and followup visits achieved sustained virological reponse. 
Demography and laboratory parameters

\begin{tabular}{|c|c|c|c|c|c|c|c|c|c|}
\hline No & Sex & $\begin{array}{c}\text { Age } \\
(15) \\
y r\end{array}$ & $\begin{array}{l}\text { APRI } \\
\text { score } \\
(0.9)\end{array}$ & $\begin{array}{c}\text { HB } \\
(12.1) \\
\text { g/dl }\end{array}$ & $\begin{array}{c}\text { TLC } \\
(8.6) \\
\text { X10 } \\
\text { IL }\end{array}$ & $\begin{array}{c}\text { PLT } \\
(425) \\
\times 10^{3} \mathrm{IL}\end{array}$ & $\begin{array}{c}\text { AST } \\
(30) \\
\text { Iu/ml }\end{array}$ & $\begin{array}{c}\text { ALT } \\
\text { (31) } \\
\text { Iu/ml }\end{array}$ & $\begin{array}{c}\text { Alb } \\
(4.7) \\
\text { Gm/dl }\end{array}$ \\
\hline 1 & $\mathrm{M}$ & 15 & 0.8 & 13 & 11 & 451 & 23 & 23 & 4.4 \\
\hline 2 & $\mathrm{M}$ & 12 & 0.6 & 12.5 & 7.7 & 336 & 33 & 65 & 4.5 \\
\hline 3 & $\mathrm{M}$ & 12 & 1.1 & 12.8 & 8.6 & 556 & 41 & 34 & 5 \\
\hline 4 & $\mathrm{M}$ & 16 & 1 & 13.1 & 7.6 & 452 & 25 & 31 & 5.1 \\
\hline 5 & $\mathrm{~F}$ & 17 & 0.9 & 13.4 & 6.6 & 346 & 35 & 24 & 5.3 \\
\hline 6 & $\mathrm{~F}$ & 17 & 0.8 & 11.8 & 10.3 & 543 & 21 & 32 & 4.9 \\
\hline 7 & $\mathrm{M}$ & 15 & 0.6 & 12 & 9.5 & 367 & 26 & 16 & 4.9 \\
\hline 8 & $\mathrm{M}$ & 15 & 1 & 13.3 & 9.4 & 365 & 27 & 18 & 4.8 \\
\hline 9 & $\mathrm{M}$ & 13 & 1.2 & 12.5 & 7.4 & 421 & 37 & 19 & 4.6 \\
\hline 10 & $\mathrm{M}$ & 14 & 1.1 & 12.8 & 6.4 & 367 & 15 & 18 & 4.2 \\
\hline 11 & $\mathrm{~F}$ & 14 & 0.5 & 12.5 & 7.7 & 432 & 41 & 33 & 4.5 \\
\hline 12 & $\mathrm{M}$ & 17 & 1.3 & 12.9 & 8.8 & 426 & 51 & 32 & 5 \\
\hline 13 & $\mathrm{M}$ & 15 & 1 & 13 & 6.5 & 438 & 23 & 52 & 5.2 \\
\hline 14 & $\mathrm{M}$ & 16 & 0.9 & 13.7 & 7.7 & 326 & 42 & 33 & 5.1 \\
\hline 15 & $\mathrm{~F}$ & 14 & 1.5 & 13.4 & 10.1 & 334 & 26 & 19 & 5.5 \\
\hline 16 & $\mathrm{~F}$ & 16 & 1.2 & 12.9 & 11.3 & 476 & 35 & 17 & 4.4 \\
\hline 17 & $\mathrm{~F}$ & 17 & 1 & 12 & 10.3 & 451 & 21 & 25 & 4.6 \\
\hline 18 & M & 16 & 1.1 & 11.9 & 9.3 & 315 & 33 & 36 & 4.3 \\
\hline 19 & M & 15 & 0.5 & 13.5 & 7.7 & 354 & 28 & 31 & 5.5 \\
\hline 20 & $\mathrm{M}$ & 17 & 0.8 & 13.1 & 8.9 & 541 & 23 & 43 & 4.1 \\
\hline 21 & $\mathrm{M}$ & 13 & 1.1 & 12.5 & 7.2 & 418 & 27 & 44 & 4.2 \\
\hline 22 & $\mathrm{M}$ & 15 & 1 & 12.7 & 9.9 & 521 & 44 & 26 & 4.4 \\
\hline 23 & M & 16 & 0.7 & 12.8 & 8.4 & 548 & 23 & 39 & 4.8 \\
\hline
\end{tabular}

Table 1: AST to platelet ratio (APRI), hemoglobin (HB), total leucocyte count (TLC), platelet (PLT), alanine aminotransferase (ALT), aspartate amino transferase (AST), albumin (ALB).

\begin{tabular}{|l|c|c|c|c|c|}
\hline $\begin{array}{l}\text { BIL (o.8) } \\
\mathbf{m g} / \mathbf{d l}\end{array}$ & $\begin{array}{c}\text { CR (0.5) } \\
\mathbf{m g} / \mathbf{d l}\end{array}$ & $\begin{array}{c}\text { HCV RNA at } \\
\text { start }\end{array}$ & $\begin{array}{c}\text { HCV RNA at 12 } \\
\text { weeks (ETR) }\end{array}$ & $\begin{array}{c}\text { HCV RNA at 24 } \\
\text { weeks (SVR) }\end{array}$ & Side effects \\
\hline 1.1 & 0.3 & positive & negative & negative & nil \\
\hline 0.4 & 0.4 & positive & negative & negative & fatigue \\
\hline 0.5 & 0.2 & positive & negative & negative & abd pain \\
\hline 0.6 & 0.5 & positive & negative & negative & nil \\
\hline 0.7 & 0.6 & positive & negative & negative & headache \\
\hline 1.2 & 1 & positive & negative & negative & fatigue \\
\hline 1 & 0.5 & positive & negative & negative & nil \\
\hline 1.1 & 0,8 & positive & negative & negative & nil \\
\hline
\end{tabular}




\begin{tabular}{|l|l|l|l|l|c|}
\hline 0.9 & 0.6 & positive & negative & negative & headache \\
\hline 0.8 & 0.6 & positive & negative & negative & nil \\
\hline 0.8 & 0.9 & positive & negative & negative & nil \\
\hline 0.3 & 0.5 & positive & negative & negative & diarrhea \\
\hline 1.1 & 0.4 & positive & negative & negative & fatigue \\
\hline 1.2 & 0.6 & positive & negative & negative & nil \\
\hline 1 & 0.4 & positive & negative & negative & nil \\
\hline 0,8 & 0.8 & positive & negative & negative & nil \\
\hline 0.7 & 0.6 & positive & negative & negative & nil \\
\hline 0.5 & 0.4 & positive & negative & negative & nil \\
\hline 1 & 0.7 & positive & negative & negative & diarrhea \\
\hline 1.2 & 0.7 & positive & negative & negative & nil \\
\hline 1 & 0.3 & positive & negative & negative & nil \\
\hline 0.7 & 0.8 & positive & negative & negative & nil \\
\hline
\end{tabular}

Table 2: Bilirubin (BIL), creatinine (CR).

\section{Discussion}

HCV is under spotlight all over the world after approval of effective treatment options in form of oral therapy with minimal side effect profile. In country like Pakistan which is having one of the highest prevalence of HCV in the world, there is indeed a great need of affective treatment strategies and options which should easily accessible as well as at low cost too, in order to achieve the goal of eradication of WHO to eradicate HCV till 2030.

Despite the availability of so many interferon free treatment regimens recommended in EASL/AASLD guidelines for the treatment of chronic HCV infected adults, yet, so far, there is only one option (SOF/ribavirin) recommended in guidelines for those below 18 years old in genotypes 2 and 3. Keeping the suboptimal response of this option specially in genotype 3 and side effects of ribavirin, More options that are pan genotypic, with low costs, easily available and short duration are highly needed for age group $[12,13]$. This study will help us solving this issue as sof/velpa is pan genotypic and highly efficacious.

This study is on the earliest study to our knowledge to be published to demonstrate the efficacy of sob/velpa combination in pediatric group of patients in genotype 3. Our results demonstrated that this treatment option is without Side effects and highly efficacious in the management of HCV from 12 to 18 years old, with high tolerability, no serious adverse effects and no treatment virologic failures. The SVR12 rate by ITT analysis was $23 / 26$. The 3 patient after achieving end treatment response, were lost to follow-up. There was no effect on liver enzymes as well as no adverse effects like hematological or kidney functions were demonstrated in this study.

There is great need to treat this age group as there are studies that shows that complication from HCV can occur in this age too, such as portal hypertension, ascites, variceal bleeding, hepatorenal syndrome, encephalopathy and liver cancer. Complications in children even below 4 year old has been reported in literature. This infection in children has affect both on quality of life and cognitive functioning. An infected person can also be a source of transmission to others in community. Due to these circumstances we decided to treat and eradicate HCV and not to wait until adolescent [11]. The excellent rate of virus eradication in this age can be due to the limited period of disease activity in body, with minimal chances of fibrosis, cirrhosis and end stage liver disease as studies shows that DAAs are more affective when they are started early before the complications develop like cirrhosis.

This is just a pilot study with limited number of patients and will be followed shortly by a full report on large numbers after completion of a multicenter study in this age which will definitely and con- 
clusively provide a more conclusive confirmation of sofosbuvir/ velpetasvir $(400+100 \mathrm{mg})$ regimen in this age group.

\section{Conclusion}

Sofosbuvir/velptasvir $(400+100 \mathrm{mg})$ combination is very safe and highly efficacious treatment option having excellent rate of sustain virological response, more than $95 \%$ in paedriatic age 12 to 18 -year patients having hepatitis $C$ genotype 3 with negligible side effects. Our pilot results should be a platform for larger and multicenter studies on this infection having genotype 3 in this age group.

\section{Bibliography}

1. y K., et al. "The safety and effectiveness of ledipasvir-sofosbuvir in adolescents 12-17 years old with hepatitis $\mathrm{C}$ virus genotype 1 infection". Hepatology 66 (2017): 371-378.

2. Polaris Observatory HCV Collaborators. "Global prevalence and genotype distribution of hepatitis $\mathrm{C}$ virus infection in 2015: a modelling study". The Lancet Gastroenterology and Hepatology 2 (2017): 161-176.

3. El-Shabrawi MH and Kamal NM. "Burden of pediatric hepatitis C”. World Journal of Gastroenterology 19 (2013): 7880-7888.

4. Delgado-Borrego A., et al. "Expected and actual case ascertainment and treatment rates for children infected with hepatitis $\mathrm{C}$ in Florida and the United States: epidemiologic evidence from state wide and nationwide surveys". Journal of Paediatrics 161 (2012): 915-921.

5. Raynes-Greenow C., et al. "Childhood hepatitis C virus infection: an Australian national surveillance study of incident cases over five years". Journal of Paediatrics and Child Health 51 (2015): 1115-1120.

6. Jadoon SA., et al. "Treatment of chronic hepatitis-C with standard interferon and ribavirin". Journal of Ayub Medical College Abbottabad 26.2 (2014): 212-215.

7. Gonzalez-Peralta RP., et al. "Hepatocellular carcinoma in 2 young adolescents with chronic hepatitis C". Journal of Pediatric Gastroenterology and Nutrition 48 (2009): 630-635.

8. Mohan P., et al. "Evaluating progression of liver disease from repeat liver biopsies in children with chronic hepatitis C: a retrospective study". Hepatology 58 (2013): 1580-1586.
9. Castellino S., et al. "The epidemiology of chronic hepatitis C infection in survivors of childhood cancer: an update of the St Jude Children's Research Hospital hepatitis C seropositive cohort". Blood 103 (2004): 2460-2466.

10. Balistreri WF., et al. "Is 3 the new 1:perspectives on virology, natural history and treatment for hepatitis C genotype 3". Journal of Viral Hepatitis 20 (2013): 669-77.

11. Quires JE and Balistreri WF. "Hepatitis $C$ virus infection in children and adolescents”. Hep comm. 1 (2017): 87-98.

12. Wirth S., et al. "Sofosbuvir and ribavirin in adolescents 12-17 years old with hepatitis C virus genotype 2 or 3 infection". Hepatology (Baltimore, Md.). (2017).

13. Indolfi G., et al. "Treatment of Chronic Hepatitis C Virus Infection in Children. A Position Paper by the Hepatology Committee of European Society of Paediatric Gastroenterology, Hepatology and Nutrition". Journal of Pediatric Gastroenterology and Nutrition 66 (2018): 505-515.

\section{Volume 2 Issue 8 October 2019 (C) All rights are reserved by Jawad Khan and Saira Nasr Malik.}

Citation: Jawad Khan and Saira Nasr Malik. "Efficacy of Sofosbuvir Velpatsavir Combination in HCV Genotype 3 Infected Children of Age between 12 to 18 Years". Acta Scientific Gastrointestinal Disorders 2.10 (2019): 02-07. 